\title{
Article \\ Stressful Life Events and Late-Life Cognitive Function in Community-Dwelling Chinese Older Adults: Findings from a Population-Based Cohort Study in the Greater Chicago Area
}

\author{
Gabriella C. Dong ${ }^{1}(\mathbb{D})$ and Mengting $\mathrm{Li}^{2,3}, * \mathbb{C}$ \\ 1 Princeton High School, Princeton, NJ 08540, USA; gabrielladong@gmail.com \\ 2 Institute for Health, Health Care Policy and Aging Research, Rutgers, The State University of New Jersey, \\ New Brunswick, NJ 08901, USA \\ 3 School of Nursing, Rutgers, The State University of New Jersey, Newark, NJ 07102, USA \\ * Correspondence: mengting.li@rutgers.edu
}

Citation: Dong, G.C.; Li, M. Stressful Life Events and Late-Life Cognitive Function in Community-Dwelling Chinese Older Adults: Findings from a Population-Based Cohort Study in the Greater Chicago Area. J. Ageing Longev. 2021, 1, 24-35. https:// doi.org/10.3390/jal1010004

Academic Editor: Notger G. Müller

Received: 4 August 2021

Accepted: 13 September 2021

Published: 27 September 2021

Publisher's Note: MDPI stays neutral with regard to jurisdictional claims in published maps and institutional affiliations.

Copyright: () 2021 by the authors. Licensee MDPI, Basel, Switzerland. This article is an open access article distributed under the terms and conditions of the Creative Commons Attribution (CC BY) license (https:// creativecommons.org/licenses/by/ $4.0 /)$.

\begin{abstract}
The majority of studies on stressful life events focus on posttraumatic stress disorder and depression, while less is known about whether the cumulative exposure to stressful life events over the life course will deteriorate cognitive function. This study aims to investigate the association between lifetime stressful life events and cognitive function in an immigrant population. The data were drawn from the Population Study of Chinese Elderly in Chicago (PINE). Face-to-face interviews were conducted with a sample of 3125 U.S. Chinese older adults in 2017-2019. Twelve types of stressful life events were assessed: physical assault, residential fires, sexual assault, miscarriage, abortion, imprisonment, being falsely accused, divorce, death of a loved one, being robbed, experiencing cancer, and being homeless. Cognitive function was measured through global cognition, episodic memory, working memory, processing speed, and Mini-Mental State Examination (MMSE). Linear regression was performed. Older adults who experienced a higher number of life events were associated with higher global cognition $(b=0.11, \mathrm{SE}=0.01, p<0.001)$, episodic memory $(\mathrm{b}=0.14$, $\mathrm{SE}=0.02, p<0.001)$, working memory $(\mathrm{b}=0.17, \mathrm{SE}=0.03, p<0.001)$, processing speed $(\mathrm{b}=1.92$, $\mathrm{SE}=0.18, p<0.001)$, and MMSE $(\mathrm{b}=0.29, \mathrm{SE}=0.07, p<0.001)$, while controlling for age, gender, income, education, medical comorbidities, ADL, and depressive symptoms. In contrast with earlier studies, we identified the positive relationships between aggregate and individual life events and cognition. Older adults who had prior experience with stressful life events could demonstrate an advantage over those without such experiences. In addition, older adults who experienced life event(s) during adulthood and old age are associated with higher cognitive function. Further studies could investigate how individuals respond to stressful life events and how the underlying resilience mechanism would promote cognitive function.
\end{abstract}

Keywords: life events; cognitive function; immigrant

\section{Introduction}

Individuals experience many stressful life events over the entire life course. Existing studies mainly focus on the experience of life events in children and youth [1]. Meanwhile, some studies investigate the relationships between life events and health among older adults but only examine life events occurring in a specific life stage (e.g., childhood adversity (ACES), recent life events) [2-7]. A few studies examine the impact of cumulative life events over the life course on late-life physical and mental health, while little is known about their impact on late-life cognition [8,9].

Existing research on the effect of stressful life events on cognition has been focused on the cumulative and differential effects of stressful life events. Some studies have found that aggregate stressful life events have a negative impact on cognitive function [5,10], while other studies did not find a significant association [4,11]. In terms of individual stressful 
life events, bereavement, the experiences of having a crime committed against a participant, and serious conflicts showed a negative impact on cognitive function [4,11-13], whereas an experience of an injury or illness of a friend in the past year positively impacted episodic memory, psychomotor speed, and attention [11].

The conflicting findings in existing studies might be attributed to not only the type of life events but also the timing of the life event. The age at which a life event is experienced could have varying effects on cognitive function. Cognitive development occurs in early childhood, while old age is a key transitional period that is coupled with cognitive decline. Studies have shown that during both early childhood and old age the brain is particularly sensitive to stress, probably because it undergoes such important changes during these periods [14]. Meanwhile, midlife hypertension, midlife obesity, and midlife hearing loss are associated with higher dementia risks [15].

Exposure to stressful life events in different life stages might have negative impacts on cognitive function. Furthermore, life events might disproportionately affect health across racial and ethnic groups due to specific cultural norms and values and thus impact varying coping strategies [16]. Asian Americans are the fastest-growing ethnic minority population in the United States. Chinese Americans comprise the largest group of Asian Americans. To fill research gaps, this study aims to (1) investigate the association between aggregate and individual life events and cognitive function and (2) examine the relationship between the stage of life at which life events are experienced and cognitive function among older Chinese Americans.

\section{Materials and Methods}

\subsection{Sample}

The PINE Study is a community-engaged, population-based panel study of older Chinese Americans aged 60 and above in the Greater Chicago area [17]. Beginning in 2011, the PINE study used culturally appropriate community recruitment strategies guided by a community-based participatory research (CBPR) approach to ensure adequate community participation [18-20]. In-person interviews were conducted by trained bicultural and bilingual interviewers at the participants' homes. Participants were interviewed in their preferred language and dialect, either Chinese (Mandarin, Taishanese, or Cantonese) or English. In a comparison between the PINE study, the 2010 US Census data, and the 2012 Random Block Census study, the results showed that the PINE sample has no significant differences in sociodemographic and socioeconomic characteristics among these studies [21]. This study utilized PINE T4 data collected from 2017-2019 with a sample size of 3125. The study was approved by the institutional review board at Rush University Medical Center in Chicago, Illinois. Written informed consent was obtained from all participants.

\subsection{Measurements}

\subsubsection{Cognitive Function}

Global cognition and three cognitive domains were assessed: episodic memory, working memory, and processing speed. Episodic memory was measured by immediate recall (EBMT) and delayed recall (EBDR) [22]. The raw scores of EBMT and EBDR were converted to $Z$ scores and then averaged to form a single episodic memory score. Working memory was tested by the Digit Span Backwards assessment (DB) [23]. Processing speed was evaluated by the 11-item Symbol Digit Modalities Test (SDMT) [24]. The Mini-Mental State Examination (MMSE) was used to study general cognition [25,26]. Z scores of the five cognitive tests were averaged to generate a global cognition score.

\subsubsection{Life Events}

Twelve types were assessed: death of a loved one, being robbed, physical assault, residential fire, divorce, cancer, false accusation of a crime, homelessness, sexual assault, imprisonment, miscarriage, and abortion. The life events evaluated in our study are adopted 
from the traumatic life events questionnaire [27] and Harvard Trauma Questionnaire [28]. The participants were asked if they experienced each of the twelve life events. The total number of life events participants experienced were summed up to evaluate the aggregate number of life events. The participants were also asked when the event occurred in their lives: childhood (age <20), adulthood (age 20-59), and old age (age 60+).

\subsubsection{Covariates}

Covariates included demographic factors (age and gender), social-economic status (years of education completed and income), and health-related variables (medical conditions, physical function, and depressive symptoms). Annual personal income was assessed on a scale from one to ten $(1=\$ 0-\$ 4999$ to $10=\$ 75,000$ and over). Medical comorbidities were evaluated by the number of diseases a participant has, including heart disease, stroke, cancer, high cholesterol, high blood pressure, diabetes, fractured hip, thyroid disease, and osteoarthritis. Physical function was measured by activities of daily living (ADL) [29]. Depressive symptoms were assessed by the Patient Health Questionnaire (PHQ-9) [30]. These covariates were commonly controlled in research on life events and cognitive function $[4,5,31]$.

\subsection{Data Analysis}

The number of life events experienced, age, gender, education, income, medical comorbidities, ADL, depressive symptoms, global cognition, episodic memory, working memory, processing speed, and MMSE were presented with descriptive statistics. A descriptive table was also present to show cognitive function by the stage of life at which the life event was experienced. Linear regression models were used to evaluate the relationship between aggregate life events/individual life events/the stage of life at which the life event was experienced and cognitive function.

\section{Results}

The PINE study interviewed 3125 Chinese participants in the Greater Chicago area in 2017-2019 with a mean age of 75.33 (SD = 8.22). The mean education was 9.23 years $(\mathrm{SD}=4.89) .61 .06 \%$ of the participants were female. $81.06 \%$ of participants reported an average annual income below $\$ 10,000$.

Table 1 presents sociodemographic and health factors by the number of life events experienced. Most participants experienced 1-2 life events $(N=2033,65.18 \%)$. Older adults who experienced 5+ life events had more depressive symptoms but better global cognition, episodic memory, working memory, processing speed, and MMSE than those who experienced $0,1-2$, and 3-4 life events.

Table 2 shows descriptive data on cognitive function by the stage of life at which life event(s) were experienced. With respect to age when experiencing life events, 462 participants (14.84\%) experienced life event(s) during childhood, 1848 participants (59.34\%) experienced event(s) during their adulthood, and 1489 participants (47.80\%) experienced life event(s) during old age. Participants who experienced life event(s) during their adulthood had the highest global cognition, episodic memory, working memory, processing speed, and MMSE, followed by participants who experienced life event(s) during old age and then by those who experienced life event(s) during their childhood.

Table 3 presents the association between the number of life events and cognitive function. When covariates were taken into account, a higher number of life events experienced was associated with a higher global cognition $(b=0.11, \mathrm{SE}=0.01, p<0.001)$, episodic memory $(b=0.14, \mathrm{SE}=0.02, p<0.001)$, working memory $(b=0.17, \mathrm{SE}=0.03, p<0.001)$, processing speed $(b=1.92, \mathrm{SE}=0.18, p<0.001)$, and MMSE $(b=0.29, \mathrm{SE}=0.07, p<0.001)$. 
Table 1. Sample Characteristics by Number of Life Events.

\begin{tabular}{|c|c|c|c|c|c|c|c|c|c|c|c|c|c|}
\hline $\begin{array}{l}\text { Number of } \\
\text { Life Events }\end{array}$ & $\mathrm{N}(\%)$ & $\begin{array}{l}\text { Age, Mean } \\
\text { (SD) }\end{array}$ & Female, $\mathrm{N}(\%)$ & $\begin{array}{l}\text { Education, } \\
\text { Mean (SD) }\end{array}$ & $\begin{array}{l}\text { Income, } \\
\text { Mean (SD) }\end{array}$ & $\begin{array}{l}\text { Medical Co- } \\
\text { morbidities, } \\
\text { Mean (SD) }\end{array}$ & $\begin{array}{l}\text { ADL, Mean } \\
\text { (SD) }\end{array}$ & $\begin{array}{l}\text { Depressive } \\
\text { Symptoms, } \\
\text { Mean (SD) }\end{array}$ & $\begin{array}{c}\text { Global } \\
\text { Cognition, } \\
\text { Mean (SD) }\end{array}$ & $\begin{array}{c}\text { Episodic } \\
\text { Memory, } \\
\text { Mean (SD) }\end{array}$ & $\begin{array}{c}\text { Working } \\
\text { Memory, } \\
\text { Mean (SD) }\end{array}$ & $\begin{array}{l}\text { Processing } \\
\text { Speed, Mean } \\
\text { (SD) }\end{array}$ & $\begin{array}{c}\text { MMSE, } \\
\text { Mean (SD) }\end{array}$ \\
\hline 0 & $724(23.21)$ & 74.80 (8.12) & 376 (51.93) & 7.88 (4.17) & $2.01(0.90)$ & $1.64(1.23)$ & $0.66(3.11)$ & $1.13(2.78)$ & $-0.60(0.90)$ & $-0.72(1.07)$ & $4.30(1.88)$ & $21.99(12.04)$ & $23.04(6.27)$ \\
\hline $1-2$ & $2033(65.18)$ & $75.84(8.32)$ & $1263(62.12)$ & $9.42(5.03)$ & $2.08(1.29)$ & $2.13(1.44)$ & $0.62(2.82)$ & $2.00(3.15)$ & $-0.26(0.94)$ & $-0.29(1.10)$ & $4.84(2.32)$ & 27.91 (13.76) & $23.85(5.76)$ \\
\hline $5+$ & $32(1.03)$ & $72.24(7.45)$ & $24(75.00)$ & $11.59(5.19)$ & $2.19(1.87)$ & $2.66(1.77)$ & $0.00(0.00)$ & $3.56(4.47)$ & $0.35(0.56)$ & $0.49(0.72)$ & $5.66(2.09)$ & $35.48(9.89)$ & 26.16 \\
\hline
\end{tabular}

Table 2. Descriptive Table for Age at Which a Life Stage is Experienced and Cognitive Function.

\begin{tabular}{|c|c|c|c|c|c|c|c|}
\hline \multicolumn{2}{|c|}{ Age at Exposure to Life Events } & \multirow{2}{*}{$\begin{array}{c}\mathbf{N}(\%) \\
462(14.84)\end{array}$} & \multirow{2}{*}{$\begin{array}{c}\begin{array}{c}\text { Global Cognition, } \\
\text { Mean (SD) }\end{array} \\
-0.32(0.98)\end{array}$} & \multirow{2}{*}{$\begin{array}{c}\text { Episodic Memory, } \\
\text { Mean (SD) }\end{array}$} & \multirow{2}{*}{$\begin{array}{c}\begin{array}{c}\text { Working Memory, } \\
\text { Mean (SD) }\end{array} \\
4.82(2.47)\end{array}$} & \multirow{2}{*}{$\begin{array}{c}\begin{array}{c}\text { Processing Speed, } \\
\text { Mean (SD) }\end{array} \\
27.17(13.98)\end{array}$} & \multirow{2}{*}{$\begin{array}{c}\text { MMSE, Mean (SD) } \\
23.17(6.15)\end{array}$} \\
\hline & Yes & & & & & & \\
\hline Childhood life event & No & $2651(85.16)$ & $-0.29(0.93)$ & $-0.34(1.09)$ & $4.78(2.20)$ & $27.05(13.55)$ & $23.99(5.67)$ \\
\hline \multirow{2}{*}{ Adulthood life event } & Yes & $1848(59.34)$ & $-0.09(0.86)$ & $-0.12(1.02)$ & $5.10(2.30)$ & $30.11(13.40)$ & $24.68(4.96)$ \\
\hline & No & $1266(40.66)$ & $-0.58(0.96)$ & $-0.67(1.12)$ & $4.34(2.06)$ & $22.65(12.67)$ & $22.68(6.56)$ \\
\hline \multirow{2}{*}{ Late-life life event } & Yes & $1489(47.80)$ & $-0.26(0.95)$ & $-0.28(1.10)$ & $4.83(2.33)$ & $27.69(13.45)$ & $23.76(5.74)$ \\
\hline & No & $1626(52.20)$ & $-0.33(0.93)$ & $-0.41(1.09)$ & $4.75(2.16)$ & $26.50(13.73)$ & $23.96(5.75)$ \\
\hline
\end{tabular}

Childhood life event (age < 20); Adulthood life event (20-59); Late-life life event (age 60+). 
Table 3. Association between Number of Life Events and Cognitive Function.

\begin{tabular}{|c|c|c|c|c|}
\hline & \multicolumn{4}{|c|}{$\begin{array}{c}\text { Outcome: Global Cognition } \\
\text { b (SE) }\end{array}$} \\
\hline & Model A & Model B & Model C & Model D \\
\hline Age & $-0.05(0.00) * * *$ & $-0.04(0.00) * * *$ & $-0.03(0.00)^{* * *}$ & $-0.03(0.00) * * *$ \\
\hline Female & $-0.28(0.03) * * *$ & $-0.08(0.03) * *$ & $-0.07(0.02)^{* *}$ & $-0.06(0.02) *$ \\
\hline Education & & $0.10(0.00)^{* * *}$ & $0.09(0.00)^{* * *}$ & $0.09(0.00)^{* * *}$ \\
\hline Income & & $0.04(0.01)^{* * *}$ & $0.04(0.01)^{* * *}$ & $0.03(0.01)^{* * *}$ \\
\hline Medical comorbidities & & & $-0.01(0.01)$ & $0.00(0.01)$ \\
\hline $\mathrm{ADL}$ & & & $-0.09(0.00)^{* * *}$ & $-0.08(0.00) * * *$ \\
\hline Depressive symptoms & & & & $-0.03(0.00) * * *$ \\
\hline \multirow[t]{3}{*}{ Number of life events } & $0.20(0.01)^{* * *}$ & $0.10(0.01)^{* * *}$ & $0.10(0.01)^{* * *}$ & $0.11(0.01)^{* * *}$ \\
\hline & \multicolumn{4}{|c|}{$\begin{array}{c}\text { Outcome: Episodic Memory } \\
\text { b (SE) }\end{array}$} \\
\hline & Model A & Model B & Model C & Model D \\
\hline Age & $-0.05(0.00)^{* * *}$ & $-0.04(0.00)^{* * *}$ & $-0.03(0.00)^{* * *}$ & $-0.03(0.00) * * *$ \\
\hline Female & $-0.24(0.04)^{* * *}$ & $-0.04(0.03)$ & $-0.04(0.03)$ & $-0.03(0.03)$ \\
\hline Education & & $0.09(0.00)^{* * *}$ & $0.09(0.00)^{* * *}$ & $0.09(0.00)^{* * *}$ \\
\hline Income & & $0.02(0.01)$ & $0.02(0.01)$ & $0.02(0.01)$ \\
\hline Medical comorbidities & & & $-0.00(0.01)$ & $0.01(0.01)$ \\
\hline $\mathrm{ADL}$ & & & $-0.08(0.01)^{* * *}$ & $-0.07(0.01) * * *$ \\
\hline Depressive symptoms & & & & $-0.02(0.01) * * *$ \\
\hline \multirow[t]{3}{*}{ Number of life events } & $0.23(0.02) * * *$ & $0.13(0.02) * * *$ & $0.13(0.02) * * *$ & $0.14(0.02)^{* * *}$ \\
\hline & \multicolumn{4}{|c|}{$\begin{array}{c}\text { Outcome: Working Memory } \\
\text { b (SE) }\end{array}$} \\
\hline & Model A & Model B & Model C & Model D \\
\hline Age & $-0.06(0.00) * * *$ & $-0.05(0.00) * * *$ & $-0.04(0.00)^{* * *}$ & $-0.04(0.00)^{* * *}$ \\
\hline Female & $-0.65(0.08)^{* * *}$ & $-0.22(0.07)^{* *}$ & $-0.21(0.07)^{* *}$ & $-0.19(0.07)^{* *}$ \\
\hline Education & & $0.21(0.01)^{* * *}$ & $0.20(0.01)^{* * *}$ & $0.20(0.01)^{* * *}$ \\
\hline Income & & $0.07(0.03) * *$ & $0.08(0.03)^{* *}$ & $0.07(0.03) *$ \\
\hline Medical comorbidities & & & $0.00(0.02)$ & $0.02(0.02)$ \\
\hline ADL & & & $-0.14(0.01)^{* * *}$ & $-0.13(0.01)^{* * *}$ \\
\hline Depressive symptoms & & & & $-0.06(0.01)^{* * *}$ \\
\hline \multirow[t]{3}{*}{ Number of life events } & $0.37(0.04)^{* * *}$ & $0.15(0.03)^{* * *}$ & $0.14(0.03)^{* * *}$ & $0.17(0.03)^{* * *}$ \\
\hline & \multicolumn{4}{|c|}{$\begin{array}{l}\text { Outcome: Processing Speed } \\
\text { b (SE) }\end{array}$} \\
\hline & Model A & Model B & Model C & Model D \\
\hline Age & $-0.67(0.03) * * *$ & $-0.59(0.02)^{* * *}$ & $-0.51(0.02)^{* * *}$ & $-0.50(0.02) * * *$ \\
\hline Female & $-3.80(0.45)^{* * *}$ & $-0.99(0.39)^{* *}$ & $-0.77(0.38) *$ & $-0.68(0.38)$ \\
\hline Education & & $1.32(0.04)^{* * *}$ & $1.30(0.04)^{* * *}$ & $1.29(0.04)^{* * *}$ \\
\hline Income & & $1.12(0.15)^{* * *}$ & $1.09(0.15) * * *$ & $1.03(0.15)^{* * *}$ \\
\hline Medical comorbidities & & & $-0.50(0.13)^{* * *}$ & $-0.39(0.13) * *$ \\
\hline ADL & & & $-0.77(0.07) * * *$ & $-0.73(0.07)^{* * *}$ \\
\hline Depressive symptoms & & & & $-0.31(0.06) * * *$ \\
\hline \multirow[t]{3}{*}{ Number of life events } & $3.08(0.21)^{* * *}$ & $1.71(0.18)^{* * *}$ & $1.77(0.18)^{* * *}$ & $1.92(0.18)^{* * *}$ \\
\hline & \multicolumn{4}{|c|}{$\begin{array}{c}\text { Outcome: MMSE } \\
\text { b (SE) }\end{array}$} \\
\hline & Model A & Model B & Model C & Model D \\
\hline Age & $-0.30(0.01)^{* * *}$ & $-0.27(0.01) * * *$ & $-0.22(0.01)^{* * *}$ & $-0.21(0.01)^{* * *}$ \\
\hline Female & $-1.62(0.19)^{* * *}$ & $-0.60(0.17)^{* * *}$ & $-0.54(0.16) * * *$ & $-0.47(0.16) * *$ \\
\hline Education & & $0.48(0.02)^{* * *}$ & $0.45(0.02)^{* * *}$ & $0.44(0.02)^{* * *}$ \\
\hline Income & & $0.14(0.07) *$ & $0.15(0.06) *$ & $0.12(0.06)$ \\
\hline Medical comorbidities & & & $-0.01(0.05)$ & $0.06(0.05)$ \\
\hline $\mathrm{ADL}$ & & & $-0.71(0.03)^{* * *}$ & $-0.68(0.03)^{* * *}$ \\
\hline Depressive symptoms & & & & $-0.21(0.02)^{* * *}$ \\
\hline Number of life events & $0.73(0.09)^{* * *}$ & $0.24(0.08) * *$ & $0.19(0.07) *$ & $0.29(0.07)^{* * *}$ \\
\hline
\end{tabular}

${ }^{*} p<0.05 .{ }^{* *} p<0.01 .{ }^{* * *} p<0.001$. 
Table 4 shows the association between each life event and cognitive function. After controlling covariates, older adults who experienced residential fires were associated with better global cognition $(b=0.12, \mathrm{SE}=0.05, p<0.05)$, episodic memory $(\mathrm{b}=0.14, \mathrm{SE}=0.07$, $p<0.05)$, and working memory $(\mathrm{b}=0.49, \mathrm{SE}=0.15, p<0.01)$. Participants who experienced physical assault were associated with better global cognition $(b=0.13, \mathrm{SE}=0.05, p<0.05)$ and working memory $(b=0.41, \mathrm{SE}=0.15, p<0.01)$. Participants who have been robbed were associated with higher global cognition $(b=0.11, S E=0.03, p<0.01)$, episodic memory $(b=0.14, \mathrm{SE}=0.05, p<0.01)$, processing speed $(\mathrm{b}=1.75, \mathrm{SE}=0.55, p<0.01)$, and MMSE $(b=0.59, \mathrm{SE}=0.22, p<0.01)$. Older adults who have experienced sexual assault were associated with better global cognition $(b=0.33, S E=0.12, p<0.01)$, episodic memory $(b=0.43, \mathrm{SE}=0.16, p<0.01)$, working memory $(\mathrm{b}=0.69, \mathrm{SE}=0.34, p<0.05)$, and processing speed $(b=5.45, \mathrm{SE}=1.86, p<0.01)$. Participants who have divorced were associated with higher processing speed $(b=1.83, \mathrm{SE}=0.83, p<0.05)$. Participants who experienced the death of a loved one had higher global cognition $(b=0.22, \mathrm{SE}=0.03, p<0.001)$, episodic memory $(b=0.31, \mathrm{SE}=0.04, p<0.001)$, working memory $(b=0.28, \mathrm{SE}=0.08, p<0.001)$, and processing speed $(\mathrm{b}=4.26, \mathrm{SE}=0.40, p<0.001)$. Those who had cancer were associated with higher global cognition $(b=0.16, S E=0.05, p<0.01)$, episodic memory $(b=0.20$, $\mathrm{SE}=0.07, p<0.01)$, working memory $(\mathrm{b}=0.34, \mathrm{SE}=0.16, p<0.05)$, and processing speed $(\mathrm{b}=2.24, \mathrm{SE}=0.84, p<0.01)$. Older women who have experienced a miscarriage were associated with higher processing speed $(b=1.47, \mathrm{SE}=0.71, p<0.05)$ and MMSE $(b=0.64$, $\mathrm{SE}=0.29, p<0.05)$. Older men who have experienced an abortion were associated with higher global cognition $(b=0.20, \mathrm{SE}=0.04, p<0.001)$, episodic memory $(\mathrm{b}=0.23, \mathrm{SE}=0.05$, $p<0.001)$, working memory $(b=0.25, \mathrm{SE}=0.11, p<0.05)$, processing speed $(\mathrm{b}=3.14$, $\mathrm{SE}=0.57, p<0.001)$ and MMSE $(\mathrm{b}=0.81, \mathrm{SE}=0.23, p<0.001)$.

Table 5 depicts the association between the stage of life at which a participant experienced a life event and cognitive function. After controlling for covariates, it was found that participants who experienced life event(s) during adulthood and old age had better global cognition (adulthood: $\mathrm{b}=0.20, \mathrm{SE}=0.02, p<0.001$; old age: $\mathrm{b}=0.11, \mathrm{SE}=0.02$, $p<0.001$ ), episodic memory (adulthood: $\mathrm{b}=0.26, \mathrm{SE}=0.03, p<0.001$; old age: $\mathrm{b}=0.17$, $\mathrm{SE}=0.03, p<0.001$ ), processing speed (adulthood: $\mathrm{b}=3.63, \mathrm{SE}=0.39, p<0.001$; old age: $\mathrm{b}=1.82, \mathrm{SE}=0.37, p<0.001$ ), and MMSE (adulthood: $\mathrm{b}=0.51$, SE $=0.16, p<0.01$; old age: $\mathrm{b}=0.35, \mathrm{SE}=0.16, p<0.05)$. Those who experienced life event(s) during adulthood $(\mathrm{b}=0.24, \mathrm{SE}=0.07, p<0.001)$ were associated with better working memory.

Figure 1 shows global cognition, episodic memory, working memory, processing speed, and MMSE by the number of life events.

Table 4. Association between Each Life Event and Cognitive Function.

\begin{tabular}{|c|c|c|c|c|c|}
\hline & $\begin{array}{c}\text { Global } \\
\text { Cognition } \\
\text { b (SE) }\end{array}$ & $\begin{array}{c}\text { Episodic } \\
\text { Memory } \\
\text { b (SE) }\end{array}$ & $\begin{array}{c}\text { Working } \\
\text { Memory } \\
\text { b (SE) }\end{array}$ & $\begin{array}{l}\text { Processing } \\
\text { Speed } \\
\text { b (SE) }\end{array}$ & $\begin{array}{c}\text { MMSE } \\
\text { b (SE) }\end{array}$ \\
\hline Fire & $0.12(0.05)$ * & $0.14(0.07) *$ & $0.49(0.15) * *$ & $1.23(0.82)$ & $0.12(0.33)$ \\
\hline Physical assault & $0.13(0.05)$ * & $0.11(0.07)$ & $0.41(0.15) * *$ & $1.59(0.81)$ & $0.61(0.33)$ \\
\hline Robbery & $0.11(0.03)^{* *}$ & $0.14(0.05) * *$ & $0.01(0.10)$ & $1.75(0.55) * *$ & $0.59(0.22) * *$ \\
\hline Sexual assault & $0.33(0.12) * *$ & $0.43(0.16) * *$ & $0.69(0.34) *$ & $5.45(1.86)^{* *}$ & $0.44(0.75)$ \\
\hline Divorce & $0.04(0.05)$ & $0.07(0.07)$ & $-0.06(0.15)$ & $1.83(0.83)$ * & $-0.25(0.34)$ \\
\hline Death & $0.22(0.03)^{* * *}$ & $0.31(0.04)^{* * *}$ & $0.28(0.08)^{* * *}$ & $4.26(0.40)^{* * *}$ & $0.23(0.17)$ \\
\hline Cancer & $0.16(0.05) * *$ & $0.20(0.07) * *$ & $0.34(0.16) *$ & $2.24(0.84)^{* *}$ & $0.44(0.34)$ \\
\hline Homeless & $-0.02(0.09)$ & $0.04(0.13)$ & $-0.08(0.27)$ & $1.60(1.44)$ & $-1.08(0.60)$ \\
\hline Imprisonment & $-0.09(0.13)$ & $-0.04(0.18)$ & $-0.34(0.39)$ & $2.67(2.23)$ & $-1.20(0.86)$ \\
\hline False accusation & $0.13(0.08)$ & $0.19(0.11)$ & $0.34(0.23)$ & $1.46(1.26)$ & $0.13(0.51)$ \\
\hline Miscarriage & $0.08(0.05)$ & $0.05(0.06)$ & $0.13(0.13)$ & $1.47(0.71) *$ & $0.64(0.29) *$ \\
\hline Abortion & $0.20(0.04)^{* * *}$ & $0.23(0.05)^{* * *}$ & $0.25(0.11) *$ & $3.14(0.57)^{* * *}$ & $0.81(0.23)^{* * *}$ \\
\hline
\end{tabular}


Table 5. Association between Age at Which a Life Stage is Experienced and Cognitive Function.

\begin{tabular}{|c|c|c|c|c|}
\hline & \multicolumn{4}{|c|}{$\begin{array}{c}\text { Outcome: Global Cognition } \\
\text { b (SE) }\end{array}$} \\
\hline & Model A & Model B & Model C & Model D \\
\hline Age & $-0.05(0.00)^{* * *}$ & $-0.04(0.00)^{* * *}$ & $-0.03(0.00)^{* * *}$ & $-0.03(0.00)^{* * *}$ \\
\hline Female & $-0.26(0.03) * * *$ & $-0.07(0.03) * *$ & $-0.06(0.02) *$ & $-0.05(0.02) *$ \\
\hline Education & & $0.10(0.00)^{* * *}$ & $0.09(0.00)^{* * *}$ & $0.09(0.00)^{* * *}$ \\
\hline Income & & $0.04(0.01)^{* * *}$ & $0.04(0.01)^{* * *}$ & $0.03(0.01)^{* * *}$ \\
\hline Medical comorbidities & & & $-0.01(0.01)$ & $0.00(0.01)$ \\
\hline ADL & & & $-0.09(0.00)^{* * *}$ & $-0.08(0.00)^{* * *}$ \\
\hline Depressive symptoms & & & & $-0.03(0.00)^{* * *}$ \\
\hline Childhood life event & $-0.08(0.04)$ & $0.02(0.04)$ & $0.01(0.03)$ & $0.03(0.03)$ \\
\hline Adulthood life event & $0.39(0.03)^{* * *}$ & $0.20(0.03)^{* * *}$ & $0.19(0.03)^{* * *}$ & $0.20(0.02)^{* * *}$ \\
\hline \multirow[t]{3}{*}{ Late-life life event } & $0.18(0.03)^{* * *}$ & $0.10(0.03)^{* * *}$ & $0.10(0.02)^{* * *}$ & $0.11(0.02)^{* * *}$ \\
\hline & \multicolumn{4}{|c|}{$\begin{array}{c}\text { Outcome: Episodic Memory } \\
\text { b (SE) }\end{array}$} \\
\hline & Model A & Model B & Model C & Model D \\
\hline Age & $-0.05(0.00) * * *$ & $-0.04(0.00) * * *$ & $-0.03(0.00)^{* * *}$ & $-0.03(0.00)^{* * *}$ \\
\hline Female & $-0.23(0.04)^{* * *}$ & $-0.04(0.03)$ & $-0.03(0.03)$ & $-0.02(0.03)$ \\
\hline Education & & $0.09(0.00)^{* * *}$ & $0.09(0.00) * * *$ & $0.09(0.00)^{* * *}$ \\
\hline Income & & $0.02(0.01)$ & $0.02(0.01)$ & $0.02(0.01)$ \\
\hline Medical comorbidities & & & $-0.00(0.01)$ & $0.00(0.01)$ \\
\hline ADL & & & $-0.08(0.01)^{* * *}$ & $-0.07(0.01)^{* * *}$ \\
\hline Depressive symptoms & & & & $-0.02(0.01)^{* * *}$ \\
\hline Childhood life event & $-0.07(0.05)$ & $0.02(0.05)$ & $0.02(0.05)$ & $0.03(0.05)$ \\
\hline Adulthood life event & $0.44(0.04)^{* * *}$ & $0.26(0.03)^{* * *}$ & $0.25(0.03)^{* * *}$ & $0.26(0.03)^{* * *}$ \\
\hline \multirow[t]{3}{*}{ Late-life life event } & $0.24(0.04)^{* * *}$ & $0.16(0.03)^{* * *}$ & $0.15(0.03)^{* * *}$ & $0.17(0.03)^{* * *}$ \\
\hline & \multicolumn{4}{|c|}{$\begin{array}{c}\text { Outcome: Working Memory } \\
\text { b (SE) }\end{array}$} \\
\hline & Model A & Model B & Model C & Model D \\
\hline Age & $-0.06(0.00)^{* * *}$ & $-0.05(0.00)^{* * *}$ & $-0.04(0.00)^{* * *}$ & $-0.04(0.00)^{* * *}$ \\
\hline Female & $-0.61(0.08)^{* * *}$ & $-0.19(0.07) * *$ & $-0.18(0.07)$ * & $-0.16(0.07) *$ \\
\hline Education & & $0.21(0.01)^{* * *}$ & $0.20(0.01)^{* * *}$ & $0.20(0.01)^{* * *}$ \\
\hline Income & & $0.07(0.03) * *$ & $0.08(0.03) * *$ & $0.07(0.03) *$ \\
\hline Medical comorbidities & & & $0.01(0.02)$ & $0.03(0.02)$ \\
\hline ADL & & & $-0.14(0.01) * * *$ & $-0.13(0.01)^{* * *}$ \\
\hline Depressive symptoms & & & & $-0.05(0.01)^{* * *}$ \\
\hline Childhood life event & $-0.06(0.11)$ & $0.16(0.10)$ & $0.15(0.10)$ & $0.18(0.10)$ \\
\hline Adulthood life event & $0.67(0.08)^{* * *}$ & $0.24(0.07) * *$ & $0.22(0.07) * *$ & $0.24(0.07)^{* * *}$ \\
\hline \multirow[t]{3}{*}{ Late-life life event } & $0.21(0.08)^{* *}$ & $0.04(0.07)$ & $0.02(0.07)$ & $0.05(0.07)$ \\
\hline & \multicolumn{4}{|c|}{$\begin{array}{l}\text { Outcome: Processing Speed } \\
\text { b (SE) }\end{array}$} \\
\hline & Model A & Model B & Model C & Model D \\
\hline Age & $-0.67(0.03)^{* * *}$ & $-0.60(0.02) * * *$ & $-0.52(0.02) * * *$ & $-0.51(0.02)^{* * *}$ \\
\hline Female & $-3.56(0.44)^{* * *}$ & $-0.88(0.38) *$ & $-0.65(0.38)$ & $-0.52(0.38)$ \\
\hline Education & & $1.32(0.04)^{* * *}$ & $1.30(0.04)^{* * *}$ & $1.29(0.04)^{* * *}$ \\
\hline Income & & $1.11(0.15)^{* * *}$ & $1.07(0.15) * * *$ & $1.01(0.15)^{* * *}$ \\
\hline Medical comorbidities & & & $-0.54(0.13) * * *$ & $-0.42(0.13)^{* *}$ \\
\hline ADL & & & $-0.76(0.07) * * *$ & $-0.72(0.07)^{* * *}$ \\
\hline Depressive symptoms & & & & $-0.32(0.06)^{* * *}$ \\
\hline Childhood life event & $-0.60(0.62)$ & $0.77(0.52)$ & $0.78(0.51)$ & $0.96(0.51)$ \\
\hline Adulthood life event & $6.02(0.46)^{* * *}$ & $3.34(0.39)^{* * *}$ & $3.45(0.39) * * *$ & $3.63(0.39) * * *$ \\
\hline \multirow[t]{3}{*}{ Late-life life event } & $2.74(0.45)^{* * *}$ & $1.63(0.38)^{* * *}$ & $1.66(0.37)^{* * *}$ & $1.82(0.37)^{* * *}$ \\
\hline & \multicolumn{4}{|c|}{$\begin{array}{c}\text { Outcome: MMSE } \\
\text { b (SE) }\end{array}$} \\
\hline & Model A & Model B & Model C & Model D \\
\hline Age & $-0.30(0.01)^{* * *}$ & $-0.27(0.01) * * *$ & $-0.22(0.01) * * *$ & $-0.21(0.01)^{* * *}$ \\
\hline Female & $-1.56(0.19)^{* * *}$ & $-0.60(0.17)^{* * *}$ & $-0.54(0.16)^{* * *}$ & $-0.45(0.16)^{* *}$ \\
\hline Education & & $0.48(0.02)^{* * *}$ & $0.45(0.02)^{* * *}$ & $0.44(0.02)^{* * *}$ \\
\hline Income & & $0.14(0.07) *$ & $0.15(0.06) *$ & $0.12(0.06)$ \\
\hline Medical comorbidities & & & $-0.01(0.06)$ & $0.06(0.06)$ \\
\hline ADL & & & $-0.72(0.03)^{* * *}$ & $-0.68(0.03)^{* * *}$ \\
\hline Depressive symptoms & & & & $-0.20(0.02) * * *$ \\
\hline Childhood life event & $-0.82(0.26)^{* *}$ & $-0.33(0.24)$ & $-0.37(0.22)$ & $-0.25(0.21)$ \\
\hline Adulthood life event & $1.47(0.19) * * *$ & $0.50(0.18)^{* *}$ & $0.41(0.16)$ * & $0.51(0.16) * *$ \\
\hline Late-life life event & $0.70(0.19) * * *$ & $0.31(0.17)$ & $0.24(0.16)$ & $0.35(0.16) *$ \\
\hline
\end{tabular}

${ }^{*} p<0.05 .{ }^{* *} p<0.01$. ${ }^{* * *} p<0.001$. 


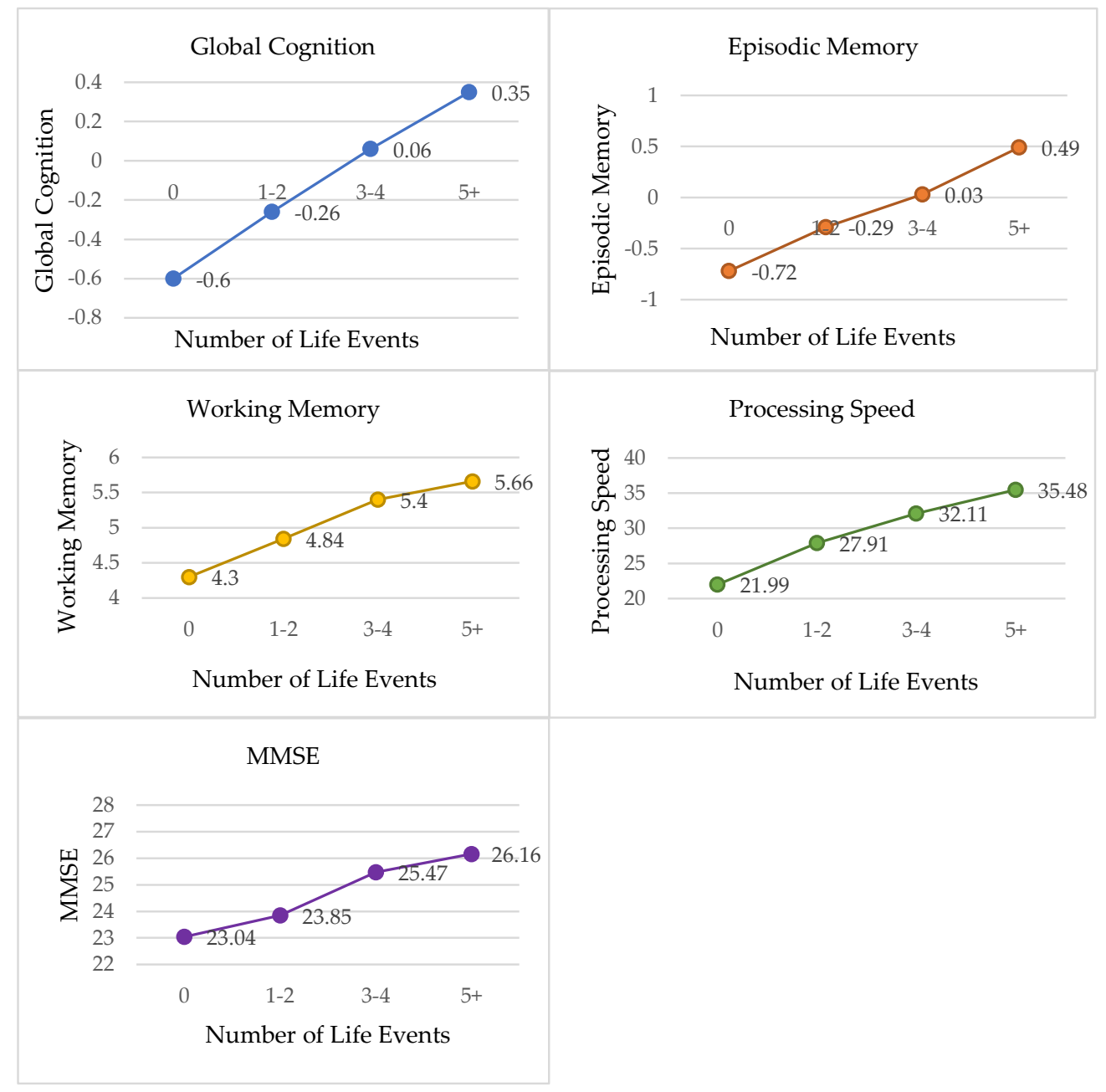

Figure 1. Cognitive Function by Number of Life Events.

\section{Discussion}

This study was among the first to examine the association between life events and cognitive function among older Chinese Americans. We found that a greater number of events were associated with higher global cognition, episodic memory, working memory, processing speed, and MMSE. In terms of individual life events, the experiences of residential fire, physical assault, robbery, sexual assault, divorce, death of a loved one, cancer, miscarriage, and abortion were associated with higher cognitive function. This study also found that experiencing life event(s) during adulthood was associated with higher global cognition, episodic memory, working memory, processing speed, and MMSE, whereas experiencing life event(s) during old age was associated with higher global cognition, episodic memory, processing speed, and MMSE.

The evidence is mixed regarding the relationship between aggregate life events and cognitive function. One study reported that a greater number of past-year life events were associated with a decline in specific areas of cognition [5]. Another study found that a higher number of stressful life events over the past three years were associated with accelerated cognitive decline among participants with mild cognitive impairment, while a higher number of life events were not associated with accelerated cognitive decline among cognitively normal participants [7]. The associations between the number of recent life events and cognition were not significant in studies conducted in Amsterdam [4] and Florida [11]. Surprisingly, we found that a greater number of life events over the life course were associated with better late-life cognitive function. A possible explanation is that life events were measured by recently occurring life events in previous research [5], 
while our study captured life events over the entire life course. Life events could have a negative impact on cognitive function in a short time immediately after the exposure but may have a salutary effect in the long run. Our findings suggested that life events might have an arousing function that stimulates cognitive function in our study population. Future studies could further examine the relationship between lifetime stressful events and cognitive function in other ethnic populations.

The impact of individual life events on cognitive function differs depending on its particular type. The negative impacts of individual life events have been well documented in the literature. Participants who have experienced the death of a child or grandchild had a higher rate of decline in general cognitive performance and learning, whereas participants who experienced the illness of a partner or relative, or serious conflicts, showed less decline in learning [4]. Other studies also identified the negative impacts of bereavement on cognitive function $[12,13]$ and experiences of having a crime committed against them on the psychomotor speed test [11]. However, the salubrious effect has been identified in an increasing number of research. Existing research reported that injury or illness of a friend [11], elder mistreatment [31], and social strain [32] were associated with better cognitive function. Our study added evidence on the positive relationship between individual life events (residential fire, physical assault, robbery, sexual assault, divorce, death of a loved one, cancer, miscarriage, and abortion) and cognitive function among older Chinese Americans.

Research on the relationship between the age at exposure to a life event and cognitive function is scarce. One prior study reported that traumatic events encountered between the ages of 18 and 30 and the ages of 31 and 64 exerted greater negative effects on late-life physical health than life events that were encountered at other ages [8]. However, our study found that this negative impact does not hold for cognitive function. On the contrary, individuals' prior exposure to life events and their lifetime cumulative experience of coping with adverse events may turn to one source of cognitive reserve in late life [33]. In addition, studies suggested that autobiographical memory is organized by culturally shared life scripts that do not include emotionally negative events while allocating important positive events to young adulthood [34,35]. This could partially explain why childhood stressful life events do not have a significant impact on the late-life cognition in our study.

The findings should be interpreted with caution. First, the experience of life events was self-reported by participants and thus may be subject to recall bias, especially given their older age. The validity of self-reported data is a controversial topic as some researchers believe recall bias makes the data seriously flawed [36], while others believe it does not because there is no clear way to detect it [37-39]. Recall bias could affect the number of lifetime stressful events as well as the age at which a life stage is experienced reported by participants. Self-reports of multiple life events could have higher risks of recall bias than self-reports of individual life events. Second, the findings of this study can not be generalized to Chinese older adults living in other geographical areas or other ethnic groups. Future studies could use random sampling to increase the sample representativeness and strengthen the generalizability of research findings. Third, participants may have experienced other life events that were not assessed in this study. Their exposures to unmeasured life events over the life course might also affect cognitive function in later life. Fourth, as this study did not assess the severity of individual life events at the time when they happened, it is important to keep in mind that the magnitude of stress a life event causes might impact its effect on cognition. Fifth, this study did not assess cognitive function during participants' childhood and adulthood; thus, we are unable to control the baseline cognition. Sixth, although the relationship between life events and cognitive function was statistically significant in this study, whether it has the clinical importance may need additional analysis on effect size. Seventh, this is a cross-sectional study. Future longitudinal studies could examine the relationship between lifetime stressful life events and cognitive decline among older adults. 
Despite these limitations, this study has important research and practical implications. This study is among the first to examine the association between life events and cognition throughout the lifespan of older Chinese immigrants. This study is highlighted by its distinction from existing literature focusing on predominately white older adults, and it provides preliminary findings for older Chinese Americans, a fast-growing ethnic population in the United States. In addition, this study examines the association between age at which a life event was encountered and late-life cognition, which is understudied in previous research. Future research could investigate the underlying resilience mechanisms through which life events have a positive effect on late-life cognition in this immigrant population. The findings of this study also serve as a foundation for future research regarding lifetime stressful life events and cognition in diverse ethnic populations. In practice, cognitive function in older age should be assessed in light of the complex array of individual and environmental factors, including stressful life events. This study could draw the attention of healthcare professionals to screen for patients' stressful life events, particularly those occurring in adulthood and old age, to better understand patients' late-life health.

\section{Conclusions}

This study examined the relationship between individual and accumulated stressful life events, age at exposure to life events, and late-life cognitive function among older Chinese Americans. Both individual and accumulated life events were associated with better cognitive function. The experience of life event(s) during adulthood and old age was associated with higher cognitive function, while the relationship between childhood life events and cognition was not significant. This study could help to inform health care professionals to assess cognitive function in older adults in light of individual and environmental factors, including stressful life events. Although the results of this study are different from prior research on recent stressful events, there was a lack of research on lifetime stressful life events and late-life cognition, and this study population is underrepresented in national studies. Future research could examine the severity of stressful life events with regards to their effect on cognition and test the relationship between positive life events and cognition in minority aging populations. Future research is needed to more precisely understand cause and effect through longitudinal studies.

Author Contributions: Conceptualization, G.C.D. and M.L.; methodology, G.C.D. and M.L.; software, M.L.; validation, G.C.D. and M.L.; formal analysis, M.L.; investigation, G.C.D. and M.L.; resources, G.C.D. and M.L.; data curation, G.C.D. and M.L.; writing—original draft preparation, G.C.D.; writing-review and editing, M.L.; visualization, G.C.D. and M.L.; supervision, M.L.; project administration, G.C.D. and M.L.; funding acquisition, M.L.; All authors have read and agreed to the published version of the manuscript.

Funding: This research was funded by Alzheimer's Association, AARG-NTF-20-684568.

Institutional Review Board Statement: The study was approved by the institutional review board at Rush University Medical Center in Chicago, IL, USA (10090203).

Informed Consent Statement: Written informed consent was obtained from all participants.

Data Availability Statement: The data presented in this study are available on request from the corresponding author.

Conflicts of Interest: The authors declare no conflict of interest.

\section{References}

1. Boals, A.; Banks, J.B. Effects of traumatic stress and perceived stress on everyday cognitive functioning. Cogn. Emot. 2012, 26, 1335-1343. [CrossRef]

2. Dannehl, K.; Rief, W.; Euteneuer, F. Childhood adversity and cognitive functioning in patients with major depression. Child Abuse Neglect. 2017, 70, 247-254. [CrossRef]

3. Amster, L.E.; Krauss, H.H. The Relationship between Life Crises and Mental Deterioration in Old Age. Int. J. Aging Hum. Dev. 1974, 5, 51-55. [CrossRef] 
4. Comijs, H.C.; van den Kommer, T.N.; Minnaar, R.W.; Penninx, B.W.; Deeg, D.J. Accumulated and differential effects of life events on cognitive decline in older persons: Depending on depression, baseline cognition, or ApoE $\varepsilon 4$ status? J. Gerontol. Ser. B Psychol. Sci. Social Sci. 2011, 66, i111-i120. [CrossRef] [PubMed]

5. Dickinson, W.J.; Potter, G.G.; Hybels, C.F.; McQuoid, D.R.; Steffens, D.C. Change in stress and social support as predictors of cognitive decline in older adults with and without depression. Int. J. Geriatr. Psychiatry 2011, 26, 1267-1274. [CrossRef] [PubMed]

6. Krause, N. Early Parental Loss, Recent Life Events, and Changes in Health among Older Adults. J. Aging Health 1998, 10, 395-421. [CrossRef]

7. Peavy, G.M.; Salmon, D.P.; Jacobson, M.W.; Hervey, A.; Gamst, A.C.; Wolfson, T.; Patterson, T.L.; Goldman, S.; Mills, P.J.; Khandrika, S.; et al. Effects of chronic stress on memory decline in cognitively normal and mildly impaired older adults. Am. J. Psychiatry 2009, 166, 1384-1391. [CrossRef] [PubMed]

8. Krause, N.; Shaw, B.A.; Cairney, J. A descriptive epidemiology of lifetime trauma and the physical health status of older adults. Psychol. Aging 2004, 19, 637. [CrossRef]

9. Shmotkin, D.; Litwin, H. Cumulative adversity and depressive symptoms among older adults in Israel: The differential roles of self-oriented versus other-oriented events of potential trauma. Soc. Psychiatry Psychiatr. Epidemiol. 2009, 44, 989-997. [CrossRef]

10. VonDras, D.D.; Powless, M.R.; Olson, A.K.; Wheeler, D.; Snudden, A.L. Differential effects of everyday stress on the episodic memory test performances of young, mid-life, and older adults. Aging Ment. Health 2005, 9, 60-70. [CrossRef]

11. Rosnick, C.B.; Small, B.J.; McEvoy, C.L.; Borenstein, A.R.; Mortimer, J.A. Negative life events and cognitive performance in a population of older adults. J. Aging Health 2007, 19, 612-629. [CrossRef]

12. Ward, L.; Mathias, J.; Hitchings, S. Relationships between bereavement and cognitive functioning in older adults. Gerontology 2007, 53, 362-372. [CrossRef]

13. Grimby, A.; Berg, S. Stressful life events and cognitive functioning in late life. Aging Clin. Exp. Res. 1995, 7, 35-39. [CrossRef] [PubMed]

14. Lupien, S.J.; McEwen, B.S.; Gunnar, M.R.; Heim, C. Effects of stress throughout the lifespan on the brain, behaviour and cognition. Nat. Rev. Neurosci. 2009, 10, 434. [CrossRef] [PubMed]

15. Livingston, G.; Sommerlad, A.; Orgeta, V.; Costafreda, S.G.; Huntley, J.; Ames, D.; Ballard, C.; Banerjee, S.; Burns, A.; CohenMansfield, J.; et al. Dementia prevention, intervention, and care. Lancet 2017, 390, 2673-2734. [CrossRef]

16. Dong, G.C.; Li, M. Potential Traumatic Events through the Life Cycle in an Immigrant Population. Geriatrics 2021, 6, 39. [CrossRef] [PubMed]

17. Dong, X. The population study of Chinese elderly in Chicago. J. Aging Health 2014, 1079-1084. [CrossRef] [PubMed]

18. Dong, X.; Wong, E.; Simon, M.A. Study design and implementation of the PINE study. J. Aging Health 2014, 26, 1085-1099. [CrossRef]

19. Dong, X.; Chang, E.-S.; Wong, E.; Simon, M. Working with culture: Lessons learned from a community-engaged project in a Chinese aging population. Aging Health 2011, 7, 529-537. [CrossRef]

20. Dong, X.; Chang, E.-S.; Simon, M.; Wong, E. Sustaining community-university partnerships: Lessons learned from a participatory research project with elderly Chinese. Gatew. Int. J. Community Res. Engagem. 2011, 4, 31-47. [CrossRef]

21. Simon, M.A.; Chang, E.-S.; Rajan, K.B.; Welch, M.J.; Dong, X. Demographic characteristics of US Chinese older adults in the greater Chicago area: Assessing the representativeness of the PINE study. J. Aging Health 2014, 26, 1100-1115. [CrossRef]

22. Albert, M.; Smith, L.A.; Scherr, P.A.; Taylor, J.O.; Evans, D.A.; Funkenstein, H.H. Use of brief cognitive tests to identify individuals in the community with clinically diagnosed Alzheimer's disease. Int. J. Neurosci. 1991, 57, 167-178. [CrossRef] [PubMed]

23. Wechsler, D. Wechsler Memory Scale-Revised; The Psychological Corporation: New York, NY, USA, 1987.

24. Smith, A. Symbol Digit Modalities Test (SDMT) Manual (Revised); Western Psychological Services: Los Angeles, CA, USA, 1982.

25. Chiu, H. Reliability and validity of the Cantonese version of mini-mental state examination-a preliminary study. J Hong Kong Coll. Psychiatr. 1994, 4, 425-428.

26. Folstein, M.F.; Folstein, S.E.; McHugh, P.R. "Mini-mental state." A practical method for grading the cognitive state of patients for the clinician. J Psychiatr. Res. 1975, 12, 189-198. [CrossRef]

27. Kubany, E.S.; Haynes, S.N.; Leisen, M.B.; Owens, J.A.; Kaplan, A.S.; Watson, S.B.; Haynes, S.N.; Owens, J.A.; Burns, K. Development and preliminary validation of a brief broad-spectrum measure of trauma exposure: The Traumatic Life Events Questionnaire. Psychol. Assess. 2000, 12, 210-224. [CrossRef] [PubMed]

28. Mollica, R.F.; Caspi-Yavin, Y. Measuring Torture and Torture-Related Symptoms. Psychol. Assess. 1991, 3, 581-587. [CrossRef]

29. Katz, S.; Akpom, C.A. A measure of primary sociobiological functions. Int. J. Health Serv. 1976, 6, 493-508. [CrossRef]

30. Spitzer, R.L.; Kroenke, K.; Williams, J.B.; Group PHQPCS. Validation and utility of a self-report version of PRIME-MD: The PHQ primary care study. JAMA 1999, 282, 1737-1744. [CrossRef]

31. Li, M.; Dong, X. Association Between Different Forms of Elder Mistreatment and Cognitive Change. J. Aging Health 2021, 33, 249-259. [CrossRef]

32. Ge, S.; Wu, B.; Bailey Jr, D.E.; Dong, X. Social support, social strain, and cognitive function among community-dwelling US Chinese older adults. J. Gerontol. Ser. A Biomed. Sci. Med. Sci. 2017, 72, S16-S21. [CrossRef]

33. Knight, B.G.; Gatz, M.; Heller, K.; Bengtson, V.L. Age and Emotional Response to the Northridge Earthquake: A Longitudinal Analysis. Psychol. Aging 2000, 15, 627-634. [CrossRef] 
34. Bemtsen, D.; Rubin, D.C. Emotionally charged autobiographical memories across the life span: The recall of happy, sad, traumatic, and involuntary memories. Psychol. Aging 2002, 17, 636-652. [CrossRef]

35. Rubin, D.C.; Berntsen, D. Life scripts help to maintain autobiographical memories of highly positive, but not highly negative, events. Mem. Cogn. 2003, 31, 1-14. [CrossRef] [PubMed]

36. Maughan, B.; Rutter, M. Retrospective reporting of childhood adversity: Issues in assessing long-term recall. J. Personal. Disord. 1997, 11, 19-33. [CrossRef] [PubMed]

37. Bernstein, D.P.; Fink, L.; Handelsman, L.; Foote, J.; Lovejoy, M.; Wenzel, K.; Sapareto, E.; Ruggiero, J. Initial reliability and validity of a new retrospective measure of child abuse and neglect. Am. J. Psychiatry 1994, 151, 1132-1136. [PubMed]

38. Goodman, G.S.; Ghetti, S.; Quas, J.A.; Edelstein, R.S.; Alexander, K.W.; Redlich, A.D.; Cordon, I.M.; Jones, D.P. A prospective study of memory for child sexual abuse: New findings relevant to the repressed-memory controversy. Psychol. Sci. 2003, 14, 113-118. [CrossRef]

39. Paivio, S.C. Stability of retrospective self-reports of child abuse and neglect before and after therapy for child abuse issues. Child Abuse Negl. 2001, 25, 1053-1068. [CrossRef] 